\title{
A SMART MONITORING SYSTEM FOR CAMPUS USING ZIGBEE WIRELESS SENSOR NETWORKS
}

\author{
Alaa Azmi Allahham, Md. Arafatur Rahman \\ Faculty of Computer Systems and Software Engineering \\ University Malaysia Pahang, 26300 - Kuantan, Pahang, Malaysia \\ alaa.allahham@gmail.com
}

\begin{abstract}
The wireless sensor networks are autonomous sensors that are distributed to monitor environmental and physical conditions and pass them across the network to other areas, which is considered one of the key elements that are used in the applications of smart cities. Therefore, this paper aims to provide a design to add more smart applications to the sanctuary and other compounds based on wireless sensor networks using ZigBee technology. The transition from reliance on the style of surveillance and controlled manually by staff to apply the principles of smart applications through wireless sensor network which provides the ability to getting all the necessary information and capabilities of controlling and monitoring are required to automatically and thus saving the time, effort, and money. The system proposed in this paper to design a smart monitoring system at the campus to control the opening and closing of the doors of many halls and the possibility of including lighting systems and appliances. The results obtained from OPNET program show that the network topology, which used within a ZigBee network vary in terms of performance, thus giving options for designers to build their network and choose technologies that suit their project.
\end{abstract}

Keywords: WSN; ZigBee; OPNET; Smart cities; Topology

\section{INTRODUCTION}

A wireless sensor networks (WSN) is a number of a network devices (called sensor node), these devices connecting together to sense and collect the information from the environment (e.g. Pressure, temperature) using radio signals, where many technologies, such as wireless communication, micro-electro-mechanical systems (MEMS) have been empowered to develop a low-cost, low-power, multifunctional sensor nodes that are small in term of size and communicate together in short distances (Song 2001, Yick, Mukherjee et al. 2008). Today, wireless sensor network starts to be deployed at a quickened pace. This new innovation is interesting with boundless potential for various application ranges including intelligent buildings, military, agricultural, emergency administration, etc. When connecting these sensors, you can assist operations of rescue by identifying the survivor's location, specifying risky areas to make the awareness of rescue team more to the overall situation. This type of network nodes has shown increasing the efficiency of rescue guarantee the safety of the rescue team. However, numerous applications of network sensor in the military, these applications aimed to limit the involvement of the personnel in the generally hazardous reconnaissance and observation missions. 
An assortment of mechanical, optical biological, magnetic, and chemical sensors sometimes appended to sensor node in order to gauge properties of the field area. Sensor node implements radio waves for wireless communication to transfer the data to a base point (e.g., an access point to a fixed infrastructure, personal handheld device, and laptop) due to the sensor node has limited. However, sensor node used the battery as prime source power source, where the second source of the power supply harvesting the power from the environment, like solar panels may be added to the node relying upon the appropriateness of the environment, where the sensor will be deployed. Contingent upon the type of sensors utilized and the application, actuators may be incorporated into the sensor (Yick, Mukherjee et al. 2008).

There are many different related works to WSN, we will describe a few example applications that have tested and deployed in the real-life environment. Macroscope of redwood (Tolle, Polastre et al. 2005) is an application used WSN to monitor and record the redwood trees in California, where sensor nodes have been deployed in order to measures relative the temperature humidity of the air, and photo-synthetically-active solar radiation. The sensor node is put at different heights of the tree, in order to help the plant biologists tracking the changes of spatial gradients in the microclimate around a redwood tree and the validation of their biological hypotheses. One of the modern applications of intelligent surveillance systems is automation and remote monitoring of houses and campuses, where Liang et al (Liang, Huang et al. 2008) used ZigBee and PSTN (Public Switched Telephone Network) technologies, to develop a wireless smart home sensor network system. While an environmental monitoring system was introduced on campus based on the distribution of ZigBee sensors in (Qian, Ma et al. 2014). As is known in road and vehicle monitoring systems, faulty tracking leads to faulty management of the system as a whole. For that, the (Lin, Li et al. 2005) used 3G technologies and GPRS communications to develop an online diagnosis and real time warning system for vehicles. In addition, many intelligent applications can be integrated into several areas, including the field of meteorological monitoring and forecasting, through the design of an environmental monitoring system, including temperature radiation, humidity, and other parameters. The (Rosiek and Batlles 2008) developed a system of data-acquisition from remote meteorological stations utilizing the mobile communication networks (more specifically, GPRS). As energy consumption is one of the major challenges for WSN (Mohsin, Bakar et al. 2012), (Sadiq, Almohammad et al. 2017) propose Energy-Efficient Cross-Layer (EECL) approach, where relies on the exploitation of interactive information from both physical and Mac layers to achieve communication between nodes with high energy efficiency.

This paper contains five sections in addition to the introduction, section 2 contains reviewed on wireless sensor networks and its important applications as well as its challenges and technologies, while section 3 will address the system design description with clarifying the designed network infrastructure. Section 4 provides technical information about the settings and parameters of the simulation program that are used in this study, while the discussion of the results and observations will be in Section 5. Finally, Section 6 contains the overall conclusions of the study.

\section{WIRELESS SENSOR NETWORK}

A wireless sensor network is a group of spatially broadcast. It can monitor and record the real situation of a location. The main function of WSN is to collect physical data 
from the environment and to send the data one or many collector nodes (Živanov, Rakić et al. 2008). Wireless Sensor Networks (WSN) consists of distributed sensor nodes that are connected wirelessly to the base station. The sensor node includes a radio transceiver through an antenna, a controller, an electronic circuit, and an energy source, usually a battery. This wireless sensor networks determine real situation environments such as wind speed, sound, humidity, pollution level, temperature and water level. A wireless sensor network contains ten or hundred sensors nodes.

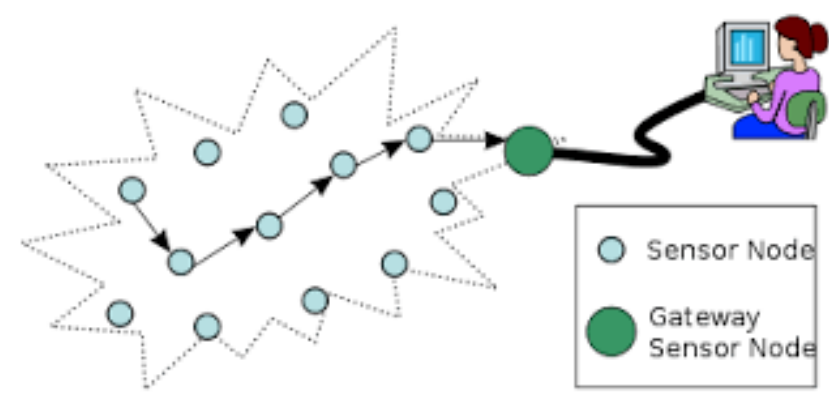

Figure 1. Wireless sensor network (Munir, Gordon-Ross et al. 2014)

\section{WSN Technologies}

\section{A) ZigBee}

ZigBee technology uses a small data rate, small energy consumption, less price, wireless networking protocol targeted toward computerization and remote control application. It is a specification for small, low power radios based on IEEE 802.15.4 2003 Wireless Personal Area Networks standard (Rahman 2014). ZigBee is expected to provide small price and less energy connect for devices and it requires battery timeconsuming as long as a number of months to a number of years. Additional, ZigBee can implement in mesh networks than Bluetooth. Coverage range of ZigBee is $10 \mathrm{~m}$ to $150 \mathrm{~m}$ with the highest data rate $250 \mathrm{kbps}$ (Rahman 2014). On the one hand, low energy spending is an important feature for wireless sensor network project.

\section{B) XBee}

XBee wireless communication standard is IEEE 802.15.4/ZigBee (Boonsawat, Ekchamanonta et al. 2010). The XBee is one part of Low- Rate Wireless Personal Area Network (LR-WPAN). XBee can provide 250kbps data transfer rate related on a Carrier Sense Multiple Access with Collision Avoidance (CSMA/CA) network (Hebel and Bricker 2010). XBee has features to data communication by using monitoring and controlling of remote devices. XBee protocol typically uses the mesh topology and it has a coverage range up to $1.6 \mathrm{~km} \mathrm{[3],} \mathrm{a} \mathrm{frequency} \mathrm{is} 2.4 \mathrm{GHz}$.

\section{C) Bluetooth}

Bluetooth is a wireless technology and it can transfer data among the various smart electronic devices. Bluetooth data transmission is smaller than the other wireless communication technology. Bluetooth technology permits hands-free headset for incoming voice calls, the ability of printing and fax and automatic synchronization of PDA. Some key features of Bluetooth technology are fewer complications, less power 
use, available at cheaper rates, robustness. Bluetooth has two connectivity topologies: the piconet and scatternet (Lee, Su et al. 2007). The standard of Bluetooth wireless connection is IEEE 802.15.1 Low Rate-Wireless Personal Area Networks (LR-WPAN) with data transfer rate is $250 \mathrm{kbps}$ compared to $1 \mathrm{Mbps}$.

\section{D) Wi-Fi}

Wireless technology (Wi-Fi) based on IEEE 802.11 specification standards for wireless local area networks (WLAN) (Nugroho and Sahroni 2014, Rahman 2014). The newest versions of Wi-Fi are version "n" and version "ac" that can offer advanced performance (Rahman 2014). Wi-Fi has the advantages of high data rate and lengthy range distance. The data rate can achieve $11 \mathrm{Mbps}$ with distances up to 100 meters however Wi-Fi requires a great power supply (Nugroho and Sahroni 2014).

Table 1. Comparison of WSN technologies

\begin{tabular}{lllll}
\hline Technology & $\begin{array}{l}\text { Data } \\
\text { Rate }\end{array}$ & Frequency & Coverage & Standard \\
\hline ZigBee & $20 \mathrm{kbps}$ & $868 \mathrm{MHz}$ in Europe & $10-150 \mathrm{~m}$ & IEEE 802.15.4 \\
& $40 \mathrm{kbps}$ & $915 \mathrm{MHz}$ in US & & \\
& $250 \mathrm{kbps}$ & $2.4 \mathrm{GHz}$ Worldwide & & \\
XBee & $250 \mathrm{kbps}$ & $2.4 \mathrm{GHz}$ & $1.6 \mathrm{~km}$ & IEEE 802.15.4 \\
Bluetooth & $1 \mathrm{Mbps}$ & $2.4 \mathrm{GHz}$ & $10 \mathrm{~m}$ & IEEE 802.15.1 \\
Wi-Fi & $150 \mathrm{Mbps}$ & $2.4-5 \mathrm{GHz}$ & $250 \mathrm{~m}$ & IEEE 802.11 \\
\hline
\end{tabular}

\section{WSN Applications}

Wireless sensor network nodes can sense surrounding, can communicate through near nodes and perform basic computation on the data being collected. Wireless sensor network supports a large range of helpful application. In addition, there are many applications in wireless sensor network such as area monitoring, health care monitoring, environmental sensing, air pollution monitoring, forest fire detection, smart home door recognition, landslide recognition, water quality sensing, natural disaster prohibition, data logging, waste water scanning and structural health monitoring (Drew and Albinger 2017, Prabhu, Balakumar et al. 2017, Prabhu, Pradeep et al. 2017, Shinghal and Srivastava 2017).

\section{WSN Challenges}

The challenges in adapting WSN system for the smart building are based on several factors such as human presence, building structure and material, electromagnetic wave broadcast, wireless network co-existence, etc (Turner, Ramli et al. 2013). Challenges and constraint of wireless sensor network include limited functional capabilities as well as size problem, power factors, node expenses, environmental factors, communication channel factors, topology management complication and node allocation, standards versus proprietary solutions and scalability concerns (Egea López 2006).

Hardware: Sensor need to fit into a tight module and a sensor node is normally of four key components and four optional components. The key components contain a power unit, a sensing unit, a processing unit, and a transceiver unit. Sensor node can 
disposable, independent, and adaptive to the location. Power Consumption: Sensor node lifetime is depending on battery existence. Environment Sensor networks often are predictable to work in an unattended style in dispersed or remote geographic locations: Nodes able to deploy in insensitive, hostile, or usually spotted environments. Such environments give increase to challenging management mechanisms.

Transmission Channels: Sensor networks frequently run in a bandwidth- and performance-constrained multi-hop wireless communications medium. These wireless communications relations function in the radio, infrared, or optical range. Some sensor devices use a single-channel RF transceiver operating, some sensor systems use a Bluetooth transceiver, other systems use Wi-Fi technology, or possibly other bands such as XBee or ZigBee.

Connection and Topology: Manage and deploy a high number of nodes in a relatively bounded environment need best techniques. More than hundreds of sensors in close closeness may be deployed in a sensor field. After the operation, topology changes may result, by cause of changes in sensor node place; power availability, dropouts, or brownouts; not working; reach ability impairments; jamming; and so on. Some sensors nodes may fail or block by reason of require power or have material spoil, ecological intrusion, that breakdown should not involve whole sensor network (Sohraby, Minoli et al. 2007).

\section{SYSTEM DESCRIPTION}

The complexes, whether residential, university campus, factories, or other, consist of a number of multi-floor buildings, where the processes of controlling and monitoring all the facilities and resources are considered one of the most basic roles. One of the issues that are pursued are the doors to the rooms and buildings to make sure it closed when not needed them and outside working hours as well as in holidays, and the same thing for the electrical resources to these halls, as well as electric appliances, such as air-conditioners.

The scenarios designed in this paper is related to the University Malaysia Pahang (UMP) Gambang campus, where is divided into two main zones separated by a distance of a number of streets estimated at 1,000 meters and the buildings in both regions contain on the laboratories and classrooms as well as staff offices as shown in figure 2. It is usually monitored and checked the doors and electricity resources in buildings manually by security staff and on a daily basis, and this requires time not less than two hours to scan the entire University buildings by the number of at least 10 security personnel, and that for one time daily only in evening. This will certainly lead to increasing the effort when the campus size increases to follow all the campus facilities. From this perspective, we need a mechanism to provide all the surveillance and control services to all campus facilities, whether buildings or the devices inside them depending on the principles of wireless sensor networks.

In this paper, we have designed a wireless sensor network (WSN) with a starmesh topology for connecting the buildings in UMP in both regions to a monitoring center depending on the ZigBee technology to monitor and control the state of the doors (open or close) inside the buildings in addition to electricity resources.

The design depends on install ZigBee sensors (ZED) on each door and connects those sensors to the ZigBee router (ZR), which will forward the data to ZigBee coordinator (ZC), where it was installed on the surface and send the data to the monitoring center via the internet as shown in figure 3. The number of ZRs and ZCs 
which will installed in the buildings depend on the ZigBee coverage area and the size of the building.

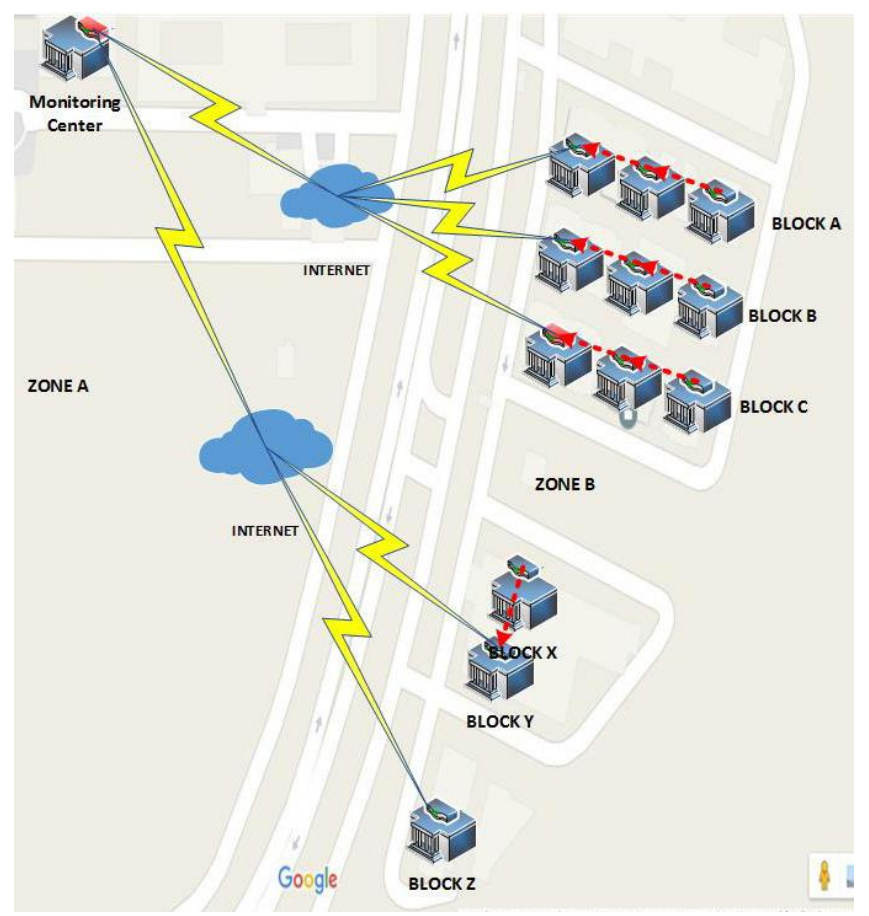

Figure 2. UMP ZigBee WSN infrastructure

Figure 3 describes the design of the network inside the building, where it was taken into account the limited coverage ranges of ZigBee devices and to give the robust to the network to prevent any interruptions may occur because of the many Obstacles. Therefore, it was installed two ZRs between the two floors in the building (where all the buildings on campus consist of two-floor maximum) to receive data from the sensors ( 3 of each floor) and then pass the data to a $\mathrm{ZC}$ that is installed on the roof of the building.

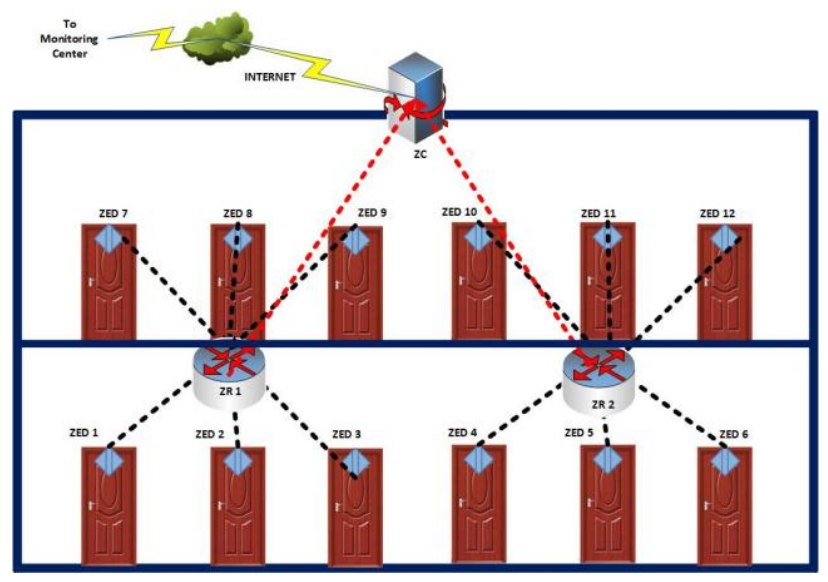

Figure 3. ZigBee WSN Building design

Figure 2 shows the designed network from a holistic perspective, where show the scheme of connecting the buildings to the monitoring center. Due to the low coverage range for ZigBee sensors, which does not exceed $20 \mathrm{~m}$ as has been adopted in this paper 
to ensure the robustness of the network, although the range may reach to $150 \mathrm{~m}$ maximum depending on what has been explained in Section 2. Therefore, the worthier way to connect the buildings from zone B to the monitoring center is via the Internet connections, where each $\mathrm{ZC}$ which used in this design contains an Ethernet output port. For adjacent buildings (Blocks A,B,C and Blocks X,Y), which the distance between them is not more than $15 \mathrm{~m}$, it will be linked with each other through mesh network between ZR which was installed on the roofs of adjacent buildings to connect them via the mesh network, after that the ZC connected to the monitoring center via the internet, where it was installed on the building closest to the monitoring center (Such as Block Z).

ZigBee is a worldwide open standard for wireless radio networks in the monitoring and control fields. The standard was developed by the ZigBee Alliance to meet the following principal needs (Boonsawat, Ekchamanonta et al. 2010) :

- Low cost.

- Ultra-low power consumption.

- Low data rate (less than $250 \mathrm{Kbps}$ ).

- Use of unlicensed radio bands.

- Cheap and easy installation.

- Flexible and easy installation.

- Integrated intelligence for network set-up and message routing.

According to the information provided in section 2, the coverage area and the throughput for the ZigBee are low in comparison to other available technologies. However, we chose the ZigBee sensors in our design, due to many reasons derived from the fact that our project requirements commensurate with the technical capabilities of ZigBee, such as we do not need high throughput because the information which will distribute in the network will be small size and it just a packets with a value 0 or 1 ( 0 means the door is open, 1 means the door is closed ). As well as comparing with the other available technologies, the ZigBee is considered the cheapest one. Furthermore, ZigBee devices can be working for years by using a single battery, in addition, it is easy to install ZigBee devices with the ability to self - configuring, as well as the ability to modify their configuration according to the requirements. There are three different devices types of ZigBee network:

- ZigBee coordinator (ZC): This kind of node can be responsible about controlling the communication data and establish links for communication..

- ZigBee Router (ZR): As well as running an application function as a router, it can act as an intermediate router, passing data from other devices.

- ZigBee End Device (ZED): Basically, this type of node is made up of different kinds of sensors and a ZigBee wireless module. In the controlling zone, according to the coverage area, the network coordinator can be received the data from all sensors throughout the network.

Recently, there are a lot of ZigBee networks would overlap each other and that would cause breakdown the node of the network. Due to this, in the design, nodes should be considered to distinguish the networks, in addition to ensure the communication between the nodes; it must examine the routing algorithm.

Figure 4 provides an example of the principle of ZigBee networks, where the network sensor and the network coordinator are the basic themes of this system in this paper. While the coordinator is connected to a computer, which has access via the internet to the server, so it does not matter where the location of the user, where the coordinator can be received the data via the internet. All the messages that transferring between the server and the network will be read and analyzed by the CPU of the network 
coordinator if the command sent by the server. All these details will be written by the main control program in the coordinator and sent via the serial ports to the ZigBee module. Here will come the responsibility of ZigBee which is sending all these messages to whole the sensors throughout the network.

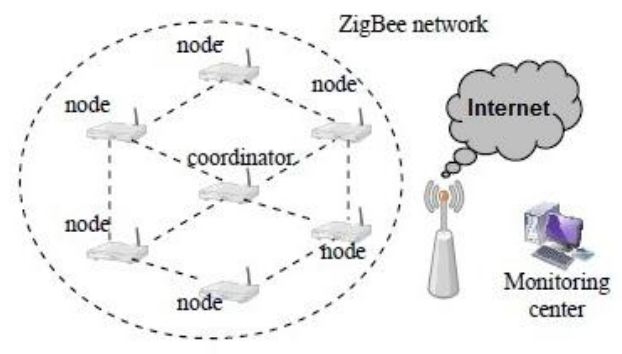

Figure 4. ZigBee WSN infrastructure (Tree 2014)

\section{SIMULATION ENVIROMENTS}

In network simulation and before starting the work, the main objective of the study must be determined clearly, because the settings and parameters of the simulator program have to be set, in order to achieve the objective. The data traffic type that will be used in the simulation program must also be specified because it plays an important role in the results that will be obtained and its accuracy. This study is based on the Opnet Modeler program, which uses explicit traffic type, where it depends on the transmitter and analyzes all the events (packet created, packet queued, the packet transmitted, etc.) on the level of each packet separately, and this gives accurate results, due to the fact that the simulation program models all affect the protocols.

\section{Main Objective}

The main objective of this research paper is to suggest a design for a wireless sensor network based on ZigBee technology, in order to provide solutions to the issue of monitoring and control of the doors and electricity resources for the buildings and halls on campus, which was discussed in section 3. Through simulation program, the proposed network will be built within the program and test it to make sure that it meets the requirements of the project, which through the test the obtained performance metrics from the simulation.

\section{Simulation Parameters}

Table 2 shows the simulation parameters that are used in all the scenarios in this study (Rahman 2014).

Table2. Simulation parameters

\begin{tabular}{ll}
\hline \multicolumn{1}{c}{ Parameters } & \multicolumn{1}{c}{ Value } \\
\hline Simulation Area & $1 \mathrm{~km} \mathrm{x} \mathrm{1} \mathrm{km}$ \\
Simulation Time & $1 \mathrm{hour}$ \\
Node movement model & Fixed \\
\hline
\end{tabular}




\begin{tabular}{lll}
\hline \multirow{3}{*}{ MAC } & ACK Wait Duration [S] & 0.05 \\
Parameters & Number of Retransmissions & 5 \\
& Minimum Backoff Exponent & 3 \\
& Maximum Number of Backoffs & 4 \\
& Channel Sensing Duration & 0.1 \\
\hline \multirow{3}{*}{ Network } & Scenario 1 & Default Tree \\
Parameters & Scenario 2 & Network \\
& & Default Mesh \\
& Data Rate & Network \\
Physical & Packet Reception-Power & Auto Calculate \\
Layer & Threshold & -85 \\
Parameters & Transmission Band & \\
& Transmit Power & $2450 \mathrm{MHz}$ \\
& Packet inter-arrival time & 0.05 \\
\hline \multirow{3}{*}{ Application } & Packet size [bits] & Constant (1) s \\
Traffic & Start Time & Constant (1024) \\
& Stop time & Uniform $(20,21)$ \\
\hline
\end{tabular}

\section{Performance Metrics}

Performance metrics is that which measured the performance of networks and analyzes the network performance and also make a comparison between different network models and systems.

1- Packet Delivery Ratio (PDR): It is the ratio of the traffic received to the traffic sent in the network.

Where:

$$
P D R=(\text { Traffic Received } / \text { Traffic Sent }) * 100 \% \ldots .(1)
$$

- Traffic Received (packets/Sec): It is a total of all packets received by router in control room.

- Traffic Sent (packets/Sec): It is a total of all packets sent by all ZigBee sensors nodes in network.

2- End-to-End Delay (secs): It is the time elapsed from the moment of creation of the packet at the source node, until received by the destination node.

3- MAC Load (bits/Sec): It is the forwarding load for each PAN to transfer the packages to the IEEE 802.15.4 Mac layer, i.e., Physical layer, by the upper layers.

4- Throughput (bits/Sec): It is a total data that have been received successfully by router in control room.

\section{Simulation Results AND ObSERVATions}

The figures 5 - 8 show the obtained results when applying the proposed design in the simulation application, where the results show the comparison and the difference between two scenarios: Tree and Mesh network, where to adjust this setting within the network parameters of the settings for ZC device as shown in Table 2.

Figure 5 shows that the PDR value for the mesh topology (approximately equal $65 \%$ ) is greater than for tree topology (approximately equal 53\%), where the traffic 
received in mesh topology is greater than in tree topology by around 5000, although the traffic sent for both of them is the same value, while figure 6 shows that the end-to-end delay in mesh topology is also less than in tree topology by approximately half. This is because of the mesh topology holding a routing table as a requirement, which gives the nodes the capability to find different alternative paths that take less time for the packets to pass from one end to another.

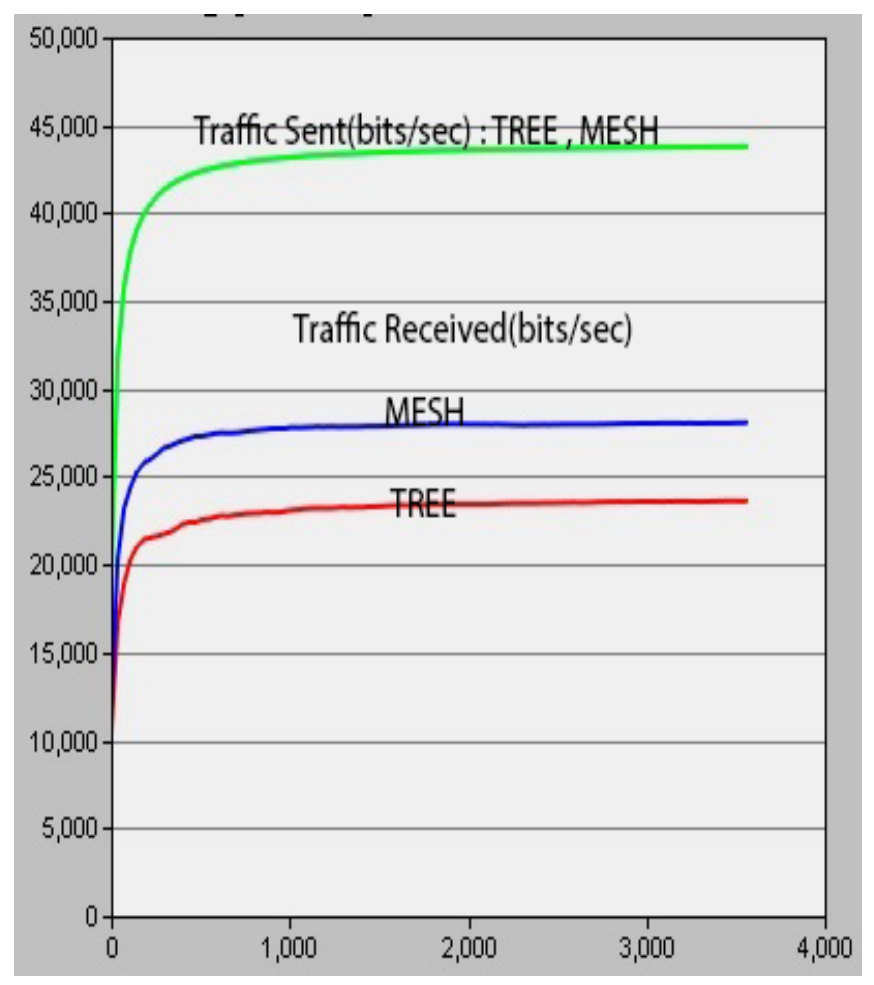

Figure 5. PDR for both ZigBee Tree and Mesh.

On the other hand, both figures 7 and 8 show that the tree topology experiences better results in both MAC load and throughput than the mesh topology. Justifying this what is shown in Figure 7 that the speed of transfer the packets from the physical layer to the upper layers is higher in the tree network, where with increasing the load, the throughput will increase. Moreover, we can justify increasing the throughput in the tree network, that the communication in tree network is larger with both ZCs and ZRs and less frequently with ZEDs, which leading to the paths which be used for data transmission to be less susceptible to collisions and congestion which increases efficiency. 


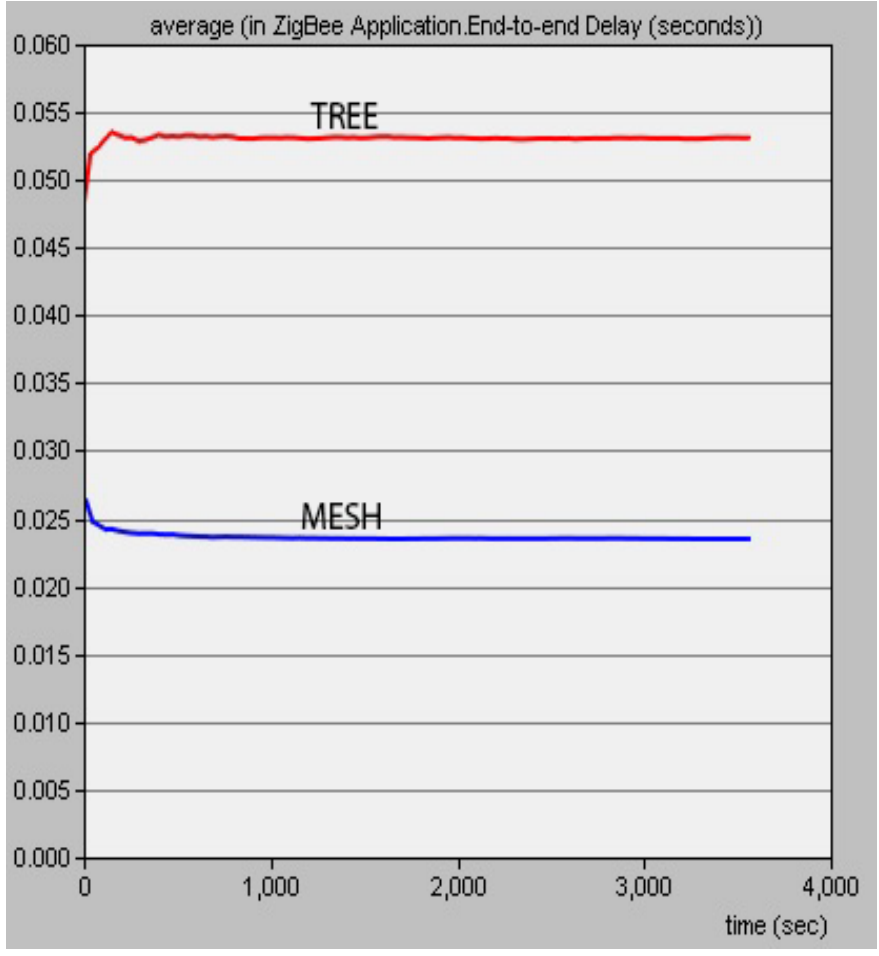

Figure 6. End-to-End Delay for both ZigBee Tree and Mesh.

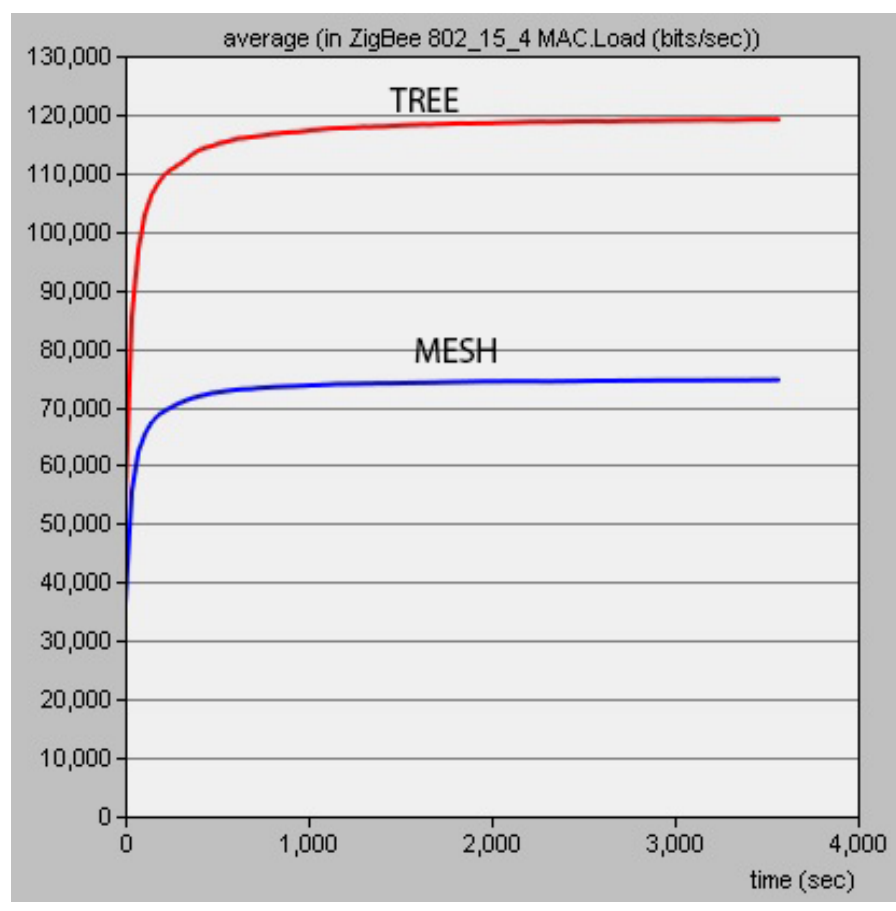

Figure 7. MAC Load for both ZigBee Tree and Mesh. 


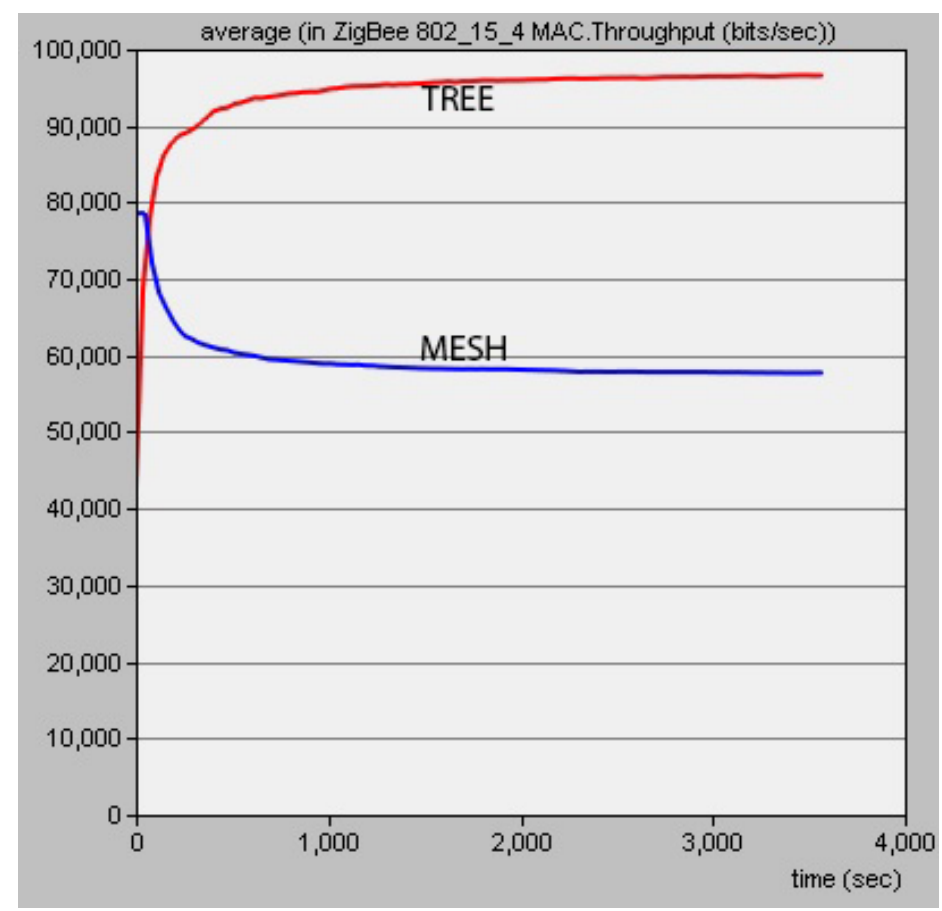

Figure 8. Throughput for both ZigBee Tree and Mesh.

\section{CONCLUSIONS}

This paper provides wireless sensor network design to build up a smart control system for the doors of the buildings and classrooms in UMP campus. The paper addresses the technologies used in wireless sensor networks, while this design was built on ZigBee technology, where the data sent from sensors via routers to coordinator and after that transmitted over the Internet to monitoring center. There are three Topologies for ZigBee networks: star, tree, mesh, where it applied both the tree and mesh topologies using Opnet modeler program. The results showed a preference of mesh in terms of PDR and delay, while the tree showed better results in terms of load and throughput.

\section{REFERENCES}

Boonsawat, V., et al. (2010). XBee wireless sensor networks for temperature monitoring. the second conference on application research and development (ECTI-CARD 2010), Chon Buri, Thailand.

Drew, B. and M. Albinger (2017). Energy harvesting wireless sensor network, Google Patents.

Egea López, E. (2006). "Simulation scalability issues in wireless sensor networks."

Hebel, M. and G. Bricker (2010). "Getting started with XBee RF modules." Parallax inc: 30.

Lee, J.-S., et al. (2007). A comparative study of wireless protocols: Bluetooth, UWB, ZigBee, and Wi-Fi. Industrial Electronics Society, 2007. IECON 2007. 33rd Annual Conference of the IEEE, IEEE. 
Liang, L., et al. (2008). Design and implementation of wireless smart-home sensor network based on ZigBee protocol. Communications, Circuits and Systems, 2008. ICCCAS 2008. International Conference on, IEEE.

Lin, C. E., et al. (2005). Development of on-line diagnostics and real time early warning system for vehicles. Sensors for Industry Conference, 2005, IEEE.

Mohsin, A. H., et al. (2012). "A survey of energy-aware routing protocols in mobile ad-hoc networks: trends and challenges." Network Protocols and Algorithms 4(2): 82-107.

Munir, A., et al. (2014). "Multi-core embedded wireless sensor networks: Architecture and applications." IEEE Transactions on Parallel and Distributed Systems 25(6): 15531562.

Nugroho, E. and A. Sahroni (2014). ZigBee and wifi network interface on Wireless Sensor Networks. Electrical Engineering and Informatics (MICEEI), 2014 Makassar International Conference on, IEEE.

Prabhu, B., et al. (2017). "Wireless Sensor Network Based Smart Environment Applications."

Prabhu, B., et al. (2017). "Military Applications of Wireless Sensor Network System."

Qian, K., et al. (2014). "A ZigBee-based building energy and environment monitoring system integrated with campus GIS." International Journal of Smart Home 8(2): 107-114.

Rahman, M. A. (2014). Enabling drone communications with WiMAX Technology. Information, Intelligence, Systems and Applications, IISA 2014, The 5th International Conference on, IEEE.

Rahman, M. A. (2014). Reliability Analysis of ZigBee Based Intra-Vehicle Wireless Sensor Networks. Nets4Cars/Nets4Trains/Nets4Aircraft.

Rosiek, S. and F. Batlles (2008). "A microcontroller-based data-acquisition system for meteorological station monitoring." Energy Conversion and Management 49(12): 3746-3754.

Sadiq, A. S., et al. (2017). An Energy-Efficient Cross-Layer approach for cloud wireless green communications. Fog and Mobile Edge Computing (FMEC), 2017 Second International Conference on, IEEE.

Shinghal, D. and N. Srivastava (2017). "Wireless sensor networks in agriculture: for potato farming."

Sohraby, K., et al. (2007). Wireless sensor networks: technology, protocols, and applications, John Wiley \& Sons.

Song, A. (2001). "Thesis Project Progress Report."

Tolle, G., et al. (2005). A macroscope in the redwoods. Proceedings of the 3rd international conference on Embedded networked sensor systems, ACM. 
Tree, S. (2014). "Wireless sensor networks." Self 1(R2): C0.

Turner, J. S., et al. (2013). The study of human movement effect on Signal Strength for indoor WSN deployment. Wireless Sensor (ICWISE), 2013 IEEE Conference on, IEEE.

Yick, J., et al. (2008). "Wireless sensor network survey." Computer networks 52(12): 22922330.

Živanov, Ž., et al. (2008). "Wireless sensor network application programming and simulation system." Computer Science and Information Systems 5(1): 109-126. 